\title{
Rates and Correlates of Alcohol Use Among Pregnant Women in Obstetrics Clinics
}

\author{
Heather A. Flynn, Sheila M. Marcus, Kristen L. Barry, and Frederic C. Blow
}

\begin{abstract}
Background: The purpose of this study was to demonstrate feasibility of screening and to identify rates and correlates of alcohol use in a large, demographically representative sample of pregnant women across a number of obstetrics clinics, extending previous studies of single or high-risk settings. Identification of harmful alcohol use during pregnancy and of associated factors is critical for the design and implementation of secondary prevention strategies.

Methods: A total of 1131 pregnant women age 18 and older were screened in the waiting areas of eight obstetrics clinics in Southeastern Michigan using a brief $(10 \mathrm{~min})$ screening questionnaire. This survey consisted of direct and indirect (TWEAK) measures of alcohol use, as well items assessing demographic characteristics, use of tobacco, and whether participants' physicians discussed alcohol use behavior with them. Women ranged in age from 18 to 46 , with a mean age of $28.7(\mathrm{SD}=5.3)$. The racial/ethnic distribution of our sample suitably reflects the various racial segments of the Michigan population.

Results: We found that $15.1 \%$ of the total sample $(n=169)$ reported any alcohol use during pregnancy, with the majority of those women reporting relatively low levels of alcohol use. One hundred and fortyseven women (13\%) scored above the cutoff on the TWEAK (i.e., above a score of 2). Based on multivariate analyses, higher risk alcohol use (defined as binge drinking or greater than one standard drink per week) during pregnancy was predicted by smoking and earlier stage of pregnancy. Caucasian race, smoking, psychological distress, and greater number of drinks during pregnancy predicted scores above a cutoff of 2 on the TWEAK.

Conclusion: This study demonstrated that screening in busy obstetrics clinics is feasible and acceptable to women and that it may be optimal to use both indirect and direct measures of alcohol use. In addition, brief assessments should be conducted throughout pregnancy and may be targeted or intensified for smokers and for women earlier in their pregnancy.
\end{abstract}

$\mathbf{P}$ RENATAL EXPOSURE TO alcohol has been shown to have detrimental effects on the developing fetus; therefore, alcohol consumption by women during pregnancy remains a major public health concern. Although fetal alcohol syndrome (FAS; the most severe outcome) is diagnosed in the infants of a relatively small percentage of mothers who are alcohol dependent (Abel, 1995), less severe developmental abnormalities may result in infants of mothers who consume lower levels of alcohol (Jacobson and Jacobson, 1994). Despite increasing public awareness of the harmful effects of drinking during pregnancy, many women consume alcohol in various degrees while pregnant. Given the potential for prevention of this public health concern, research aimed at prevention efforts is crucial.

From the Department of Psychiatry, University of Michigan Medical School, Ann Arbor, Michigan.

Received for publication March 19, 2001; accepted September 27, 2002.

Supported by NIAAA Grant 1R03AA11922-01A1.

Reprint requests: Heather A. Flynn, PhD, University, Department of Psychiatry, Substance Abuse Division, University of Michigan Medical School, 400 E. Eisenhower Pkwy, Building 2, Suite A, Ann Arbor, MI 48108-3318; Fax: 734-615-6085; E-mail: hflynn@umich.edu.

Copyright () 2003 by the Research Society on Alcoholism.

DOI: 10.1097/01.ALC.0000046595.47491.37
Community-based universal prevention efforts (Hankin, 1994) appear to have limited effectiveness in eliminating alcohol use during pregnancy for women at highest risk. Secondary or selective prevention strategies would be enhanced by effective identification of harmful alcohol use during pregnancy and of factors that are associated with prenatal drinking. Specification of characteristics of women who may be at the highest risk will aid in tailoring detection and prevention strategies (Weiner and Morse, 1996). This need is particularly compelling given evidence that at-risk women respond to preventive efforts such as supportive counseling (Larsson, 1983; Little et al., 1984; Smith et al., 1987) or more intensive counseling (Barry, 1999; Halmesmaki, 1988).

Healthcare providers who have contact with pregnant women may be valuable agents to provide effective interventions. However, many providers and medical staff do not routinely conduct systematic, effective screening (Chang et al., 1999b; Hans, 1999; Stratton et al., 1996). Screening in general medical settings (such as primary care and obstetrics clinics) is an important step in development of prevention strategies because most women in the United States will seek prenatal care at some point in their pregnancy (Brown, 1989). Thus, prenatal care visits provide an 
ideal opportunity to identify and treat mental health issues such as problem drinking and substance use disorders.

It has been suggested that alcohol consumption rates in the United States during pregnancy are increasing, with one study finding an increase from $9.1 \%$ of pregnant women reporting any alcohol use (in the past month) in 1991 to $15.9 \%$ in 1995 (Ebrahim et al., 1998). The prevalence of alcohol use during pregnancy in 1992 was estimated by an NIH report to be $18.8 \%$ (National Pregnancy and Health Survey, 1996). In a recent investigation (Kelly et al., 2001), 186 pregnant women were assessed in one university-based obstetrics clinic for current and past substance use by using a patient health questionnaire and the CAGE, a four-item alcohol screening measure assessing consequences of alcohol use (Ewing, 1984). They found that $17 \%$ of the women scored above a cutoff score of 1 on the CAGE, and 19\% reported any substance use either currently or in the past year.

Studies attempting to elucidate factors associated with alcohol use during pregnancy have yielded inconsistent findings. These studies are difficult to compare due to lack of overlap in populations and settings studied, factors assessed, and measurements used. For example, Heller et al. (1988) found that women seeking antenatal care in university-based obstetrics clinics in the United Kingdom who drank during pregnancy tended to be older, married, of higher socioeconomic status, and better educated. However, Waterson and Murray-Lyon (1989) found no difference between women who drank while pregnant and those who did not in terms of age, social class, marital status, or parity. Other studies have found significant associations between use of alcohol during pregnancy and heavy drinking prior to pregnancy (Waterson and Murray-Lyon, 1989), smoking (Day et al., 1993; Svikis et al., 1997), and use of illicit drugs (Day et al., 1993). Smith et al. (1987) examined biological, social, and behavioral factors associated with continuous drinking during pregnancy in sample of primarily African American women seeking prenatal care from a large urban hospital setting. Women who continued to drink were not found to differ from those who discontinued drinking with regard to race, income, age, marital status, parity, or use of caffeine or other drugs. These drinking groups did differ significantly on social context of drinking, chronicity of drinking, and family and medical history.

Many of these and other studies of maternal risk factors for drinking during pregnancy have been conducted in specialty (midwife centers) or high-risk clinics and with populations considered to be at highest risk, such as low income and/or minority populations (see May, 1996 for a review). Given that high-risk alcohol use occurs in women across race and socioeconomic status, it is important to study risk factors for drinking during pregnancy in other populations (Hankin et al., 2000) as well as in multiple, representative obstetrics clinics.

This study provides information on rates of alcohol use during pregnancy and factors associated with drinking dur- ing pregnancy in women screened across a number of obstetrics clinics in southeastern Michigan. We also aimed to demonstrate the feasibility of screening for alcohol use in a large number of women seeking prenatal care across several clinics. This study addresses methodological limitations found in some previous studies including sample size, sample representativeness, response rate, and use of screening instruments validated for use with women and during pregnancy.

\section{METHODS}

\section{Procedures}

All pregnant women age 18 and older were approached in the waiting areas of eight obstetrics clinics in southeastern Michigan by trained research assistants between May 1998 and July 1999. The majority of the clinic sites $(n=5)$ were university-affiliated health clinics, which serve predominantly obstetrics patients with managed care insurance plans (70.6\%) but also patients with Medicare (7.9\%), Medicaid (14.4\%), or no/unknown insurance $(7.1 \%)$. The remaining three clinics were small, private clinics in urban areas serving primarily Medicaid beneficiaries and uninsured women (no specific percentages were available from these clinic sites). These three additional clinics were chosen based on their location in urban areas and their higher number of obstetrics visits per day. All obstetrics clinics in urban areas within 45 miles of the University of Michigan were contacted, and estimates of their daily number of visits were obtained. Clinics that reported fewer than 15 prenatal care visits per day were not included for this study to maximize efficient use of research staff time. The first three clinics that met the visit criteria were included in the study. All study procedures were approved by the University of Michigan Medical School Institutional Review Board. Research assistants were blended into normal clinic operations as much as possible so as to be minimally intrusive and to simulate routine clinic screening (e.g., they sat behind the check-in desk next to clerical staff). Women were asked to complete a brief (10-15 min) screening questionnaire while they waited for their prenatal care appointments. Every English-speaking pregnant woman in the waiting area of the obstetrics clinics was approached and offered an opportunity to complete the screening survey, all of which were self-administered. The majority of women approached $(92 \%)$ agreed to complete the screening questionnaire. Confidentiality was maintained by use of study code numbers rather than names or other identifying information. Woman were told that their participation in the study was independent of their care at the clinics and that their physician and clinic staff (or anyone else other than the study investigators) would have no access to their responses. All women who were screened were provided with an educational booklet describing all aspects of a healthy pregnancy including diet, exercise, and the harmful effects of alcohol and tobacco use.

\section{Participants}

A total of 1131 pregnant women completed screening questionnaires. Women ranged in age from 18 to 46 , with a mean age of 28.7 ( $\mathrm{SD}=5.3$ ). The racial/ethnic distribution of the sample suitably reflected the various racial/ethnic segments of the southeastern Michigan population based on the 2000 U.S. Census data for the county (Census Bureau, 2000): 72\% Caucasian, $13 \%$ African American, 9.1\% Asian, 2.3\% Latino, and 0.4\% American Native. Of the women screened, $77 \%$ were married, and $72 \%$ reported having beyond a high school education ( $0.4 \%$ 8th grade or less, $6.8 \%$ completed grade $9-11$, and $20.7 \%$ completed high school). Sixty percent were working at least part-time (39.7\% working full time). Participants were recruited at various stages of pregnancy, ranging from 3 to 41 weeks $($ mean $=24.9, \mathrm{SD}=10.4$ weeks). The majority of women reported that they had partial or full custody of other children (59\%), with a mean of 0.83 children $(\mathrm{SD}=0.95)$. 
Table 1. Drinking Rates Among Women Who Reported Using Alcohol While Pregnant ( $n=169$ of 1116 Screened and Reporting Drinking Data)

\begin{tabular}{|c|c|c|c|c|c|c|c|c|}
\hline $\begin{array}{l}\text { Standard drinks } \\
\text { per week }\end{array}$ & $\%$ & $n$ & Binge episodes & $\%$ & $n$ & TWEAK $\geq 2$ & $\%$ & $n$ \\
\hline Less than 1 & 81.9 & 136 & None & 93 & 157 & No & 80.7 & 134 \\
\hline $1-2$ & 9.6 & 16 & 1 & 4.3 & 7 & Yes & 19.3 & 32 \\
\hline $2-3$ & 3.6 & 6 & 2 & 1.2 & 2 & & & \\
\hline $3-5^{a}$ & 2.4 & 4 & 3 or more & 0.6 & 1 & & & \\
\hline 5 or more & 2.4 & 4 & & & & & & \\
\hline
\end{tabular}

ns vary slightly due to missing data on items.

a The range 3-5 was chosen since there was a frequency of zero for four drinks per week.

Table 2. Intercorrelations Among Study Variables

\begin{tabular}{|c|c|c|c|c|c|c|c|c|c|c|c|}
\hline Variables & $\begin{array}{l}\text { 1. High- } \\
\text { risk } \\
\text { drinking }\end{array}$ & 2. CES-D & $\begin{array}{l}\text { 3. Weeks of } \\
\text { pregnancy }\end{array}$ & $\begin{array}{l}\text { 4. Cigarettes } \\
\text { per day }\end{array}$ & $\begin{array}{l}\text { 5. Years of } \\
\text { school }\end{array}$ & $\begin{array}{l}\text { 6. Physician } \\
\text { ask about } \\
\text { drinking } \\
\text { (yes/no) }\end{array}$ & $\begin{array}{l}\text { 7. Maternal } \\
\text { age }\end{array}$ & $\begin{array}{l}\text { 8. Marital } \\
\text { status }^{\mathrm{b}}\end{array}$ & $\begin{array}{l}\text { 9. Race/ethnic } \\
\text { group }^{c}\end{array}$ & $\begin{array}{l}\text { 10. TWEAK } \\
\text { score }\end{array}$ & 11. Parity \\
\hline 1. & $x$ & $0.07^{*}$ & 0.01 & $0.08^{\star \star}$ & 0.04 & $0.11^{\star *}$ & $0.10^{\star *}$ & -0.01 & 0.04 & $0.13^{\star *}$ & -0.02 \\
\hline 2. & & $\mathrm{x}$ & 0.03 & $0.18^{\star *}$ & $-0.28^{\star \star}$ & $0.06^{*}$ & $-0.22^{\star *}$ & $-0.25^{\star \star}$ & $-0.26^{* *}$ & $0.23^{\star *}$ & 0.03 \\
\hline 3. & & & $x$ & 0.03 & -0.01 & -0.02 & -0.02 & -0.01 & -0.00 & -0.06 & 0.05 \\
\hline 4. & & & & $x$ & $-0.35^{\star \star}$ & 0.05 & $-0.19^{\star *}$ & $-0.21^{\star \star}$ & -0.04 & $0.20^{\star \star}$ & $0.09^{\star \star}$ \\
\hline 5. & & & & & $x$ & 0.06 & $0.50^{\star \star}$ & $0.44^{\star \star}$ & $0.27^{\star \star}$ & $-0.15^{\star \star}$ & $-0.18^{\star \star}$ \\
\hline 6. & & & & & & $\mathrm{x}$ & -0.00 & -0.03 & 0.01 & $0.15^{\star \star}$ & $-0.11^{\star \star}$ \\
\hline 7. & & & & & & & $x$ & $0.33^{\star \star}$ & $0.29^{\star \star}$ & $-0.20^{* *}$ & $0.21^{\star \star}$ \\
\hline 8. & & & & & & & & $\mathrm{x}$ & $0.44^{* *}$ & $-0.09^{\star *}$ & $-0.07^{\star}$ \\
\hline 9. & & & & & & & & & $x$ & 0.02 & $-0.09^{\star \star}$ \\
\hline 10. & & & & & & & & & & $x$ & $-0.13^{\star \star}$ \\
\hline 11. & & & & & & & & & & & $x$ \\
\hline
\end{tabular}

a High-risk drinking is defined and coded as follows: $0=$ no alcohol use during pregnancy; $1=$ reported alcohol use during pregnancy is less than 1 drink per week and no binge episodes; 2 = reported $\geq 1$ drink per week or $\geq 1$ binge episode during pregnancy; ${ }^{\text {b }}$ Marital status: higher scores $=$ married or live in partner, lower values $=$ unmarried and no live-in partner; ${ }^{\mathrm{c}}$ Race/ethnic group: correlations based on comparisons of Caucasian vs. African American, with higher values $=$ Caucasian status.

${ }^{*} p<0.05 ;{ }^{* *} p<0.01$.

\section{Measures}

The screening questionnaire developed for this study consisted of items assessing demographic characteristics, health behaviors such as overall physical health, mood, exercise, and use of alcohol and tobacco during pregnancy. Questions about alcohol use were embedded within other health and lifestyle items, with potentially less threatening behaviors such as overall health, exercise, and tobacco use addressed first. Many patients are more likely to respond to questions about their alcohol consumption if placed in the broader context of health behaviors and health screening in clinical settings (Babor et al., 1987; Fleming and Barry, 1991; Fleming et al., 1997; Saunders et al., 1993). Women were asked an initial question about whether they had used any alcohol at all during their pregnancy (yes or no). Women who reported that they used any alcohol since they became pregnant were asked three follow-up questions, including (1) quantity of alcohol use ("On average, when you have had something to drink, how many drinks have you had on each occasion"), (2) frequency of alcohol use ("On average, how many days per week have you had any alcohol to drink, for example, beer, wine or any drink containing alcohol?"), and (3) binge episodes ("How often have you had five or more drinks on one occasion?"). For these items, a drink was defined for the participants as one 12-ounce beer, one 4-ounce glass of wine, and one 1-ounce shot of liquor. These items have been used in other alcohol screening studies in primary care settings (Fleming and Barry, 1991; Fleming et al., 1997). The screen also included one item asking women to indicate the number of cigarettes they have smoked each day since they became pregnant.

The screening questionnaire also included the TWEAK as an alcohol screener. Due to concerns about denial or minimization that direct questioning about alcohol use may provoke, indirect measures, such as the TWEAK, were developed. The TWEAK was designed to overcome problems with other screening measures of alcohol use consequences that have been criticized for lack of sufficient sensitivity for use with women as well as a focus on identification of severe, chronic alcohol-dependent patients who already have awareness of their alcohol problems (Russell, 1994). The TWEAK has been found to demonstrate superior sensitivity and specific- ity (compared with the CAGE or Alcohol Use Disorders Identification Test) in screening for risk drinking in women (Bradley et al., 1998) and during pregnancy (compared with the MAST or CAGE; Russell, 1994). It is a five-item measure, from which a total severity score was derived. Our instructions for the TWEAK asked women to respond based on their alcohol use behavior for the time frame of 1 year immediately before this pregnancy.

Current distress was measured by the Center for Epidemiologic Studies-Depression Scale (CES-D; Radloff, 1977). The CES-D is used widely as a screening instrument to detect depression in nonclinical populations. Items on the CES-D cover the previous 7 days and are rated on a 4-point scale. A total score is derived by summing the ratings across the 20 items. Good internal consistency (Cronbach's $\alpha=0.84$ ) has been found for the CES-D in the general population (Corcoran and Fisher, 1987), and it correlates strongly with the Beck Depression Inventory (Santor et al., 1997). A standard cut-point of 16 was used to determine elevated distress (Husaini et al., 1980).

\section{Data Analysis Plan}

Descriptive information on alcohol use during pregnancy and all other study variables was examined before we conducted any statistical analyses. Descriptions of alcohol use among the $15 \%$ of women who reported using any alcohol while pregnant ( $n=169$ of 1116 who responded to alcohol use items) are presented in Table 1. Intercorrelations among all key study variables were also examined and are presented in Table 2 . To analyze differences among various levels of alcohol use during pregnancy, three alcohol use groups were created for use in the analyses. One group consisted of women who reported no alcohol use during pregnancy $(n=$ 873). A second group (which will be referred to as the low-risk group, $n=$ 122 ) included women who reported using less than one standard drink per week and no reports of binge episodes during pregnancy. Finally, a higher risk group consisted of women who reported one or more standard drinks per week and/or at least one binge episode during pregnancy $(n=31)$. 
Table 3. Multivariate Analyses of Variance of Alcohol Use Risk Groups

\begin{tabular}{|c|c|c|c|c|c|}
\hline Variable & $\begin{array}{c}\text { No use } \\
\text { Mean }^{\mathrm{b}} \text { (SD) }\end{array}$ & $\begin{array}{l}\text { Low risk } \\
\text { Mean (SD) }\end{array}$ & $\begin{array}{l}\text { Higher risk } \\
\text { Mean (SD) }\end{array}$ & $F$ & $p$ value \\
\hline Weeks of pregnancy & $24.5(10.4)$ & $26.9(10.2)^{a}$ & $20.8(10.6)^{b}$ & 4.7 & 0.01 \\
\hline Cigarettes per day & $0.24(.74)^{\mathrm{a}}$ & $0.36(.86)^{\mathrm{a}}$ & $0.75(1.2)^{b}$ & 6.9 & 0.001 \\
\hline Marital status & & & & NS & NS \\
\hline Maternal age & $28.5(5.4)$ & $29.9(4.5)$ & $29.4(5.7)$ & 3.7 & 0.023 \\
\hline CES-D & & & & NS & NS \\
\hline Race/ethnic group & & & & NS & NS \\
\hline Parity & & & & NS & NS \\
\hline Year of school & & & & NS & NS \\
\hline
\end{tabular}

MANOVA $F(14,2030)=3.23, p<0.000$. a Alcohol use risk groups: No alcohol use $(n=873)$ : no use of alcohol during pregnancy; Low risk $(n=122)$ : less than

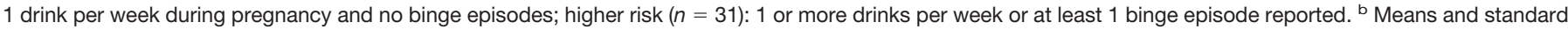

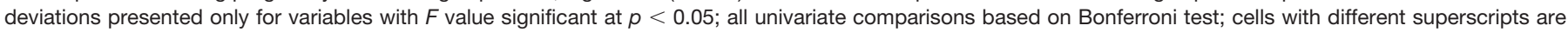
significantly different from each other, all $p s<0.05$.

Women who did not provide quantity and frequency data for alcohol use during pregnancy $(n=90)$ could not be included in analyses using these three drinking groups. Analysis of variance did not reveal any significant differences in any of the key study variables between participants who provided complete alcohol use data and those who did not.

Multivariate analysis of variance was used to examine differences among these three drinking groups in a number of variables. Demographic and health behavior variables were selected based on prior research that indicated their possible relationship to alcohol use during pregnancy: maternal age, smoking, marital status, race/ethnicity, education, parity, and weeks of pregnancy. We were also interested in the relationship between a participant's reports of whether her physician discussed alcohol use during pregnancy with her (coded dichotomously as "yes" or "no") as well as the possible influence of psychological distress (as measured by the CES-D).

Logistic regression was also conducted to examine the likelihood of scoring above the cutoff score of 2 on the TWEAK among these same study variables (maternal age, smoking, marital status, race/ethnicity, education, parity, weeks of pregnancy, physician mention of alcohol use during pregnancy, and psychological distress). Before we conducted any multivariate analyses, all data were inspected for possible violation of necessary assumptions for multivariate analyses (normality, linearity, and homoscedasticity), and no data transformations were made.

\section{RESULTS}

\section{Descriptions of Alcohol Use During Pregnancy}

Descriptive information on alcohol use behavior during pregnancy is displayed in Table 1 . As can be seen in Table $1,15.1 \%$ of the total sample of $1116(n=169)$ reported any alcohol use during pregnancy. The majority of those women reported relatively low levels of alcohol use. For example, $86.7 \%$ reported one or fewer standard drinks per week. Most women sampled (93\%) reported no binge episodes, defined as five or more standard drinks on one occasion, with $7 \%$ reporting one or more binge episodes. Of the total sample screened, 147 women $(13 \%)$ scored above the cutoff on the TWEAK. Of women in the higher risk group, $29 \%$ (10 of 34) scored above the cutoff on the TWEAK. As can be seen in Table 1, 19\% of the women who reported any alcohol use during pregnancy (32 of 166) scored above the cutoff on the TWEAK (i.e., above a score of 2).

Among women who reported any use of alcohol during pregnancy, $54.5 \%$ reported that their healthcare provider had talked with them about drinking while pregnant.
Women who used alcohol in the year before this pregnancy and reported no alcohol use during pregnancy were asked about reasons for abstaining. The most frequently reported reason $(67 \%)$ was "concern about harmful effects related to my pregnancy," followed by "didn't like the taste or effects" (16.4\%) and "health concerns" (12.6\%).

Bivariate correlations among all study variables are presented in Table 2. As can be seen, higher risk drinking was most strongly associated with TWEAK score but also had significant positive associations with smoking, CES-D score, marital status, and physician inquiry about alcohol use during pregnancy.

\section{Factors Associated With Reported Alcohol Use During Pregnancy}

As stated, multivariate analysis of variance was used to examine the presence of significant differences between the three alcohol use groups on a number of key variables. The drinking groups are defined as follows: no alcohol use during pregnancy $(n=873)$, low risk $(n=122$ defined as less than one standard drink per week and no binge episodes), and higher risk ( $n=31$, defined as one or more drinks per week and/or at least one binge episode reported). The overall model was found to be significant $[F(14,2030)=3.23, p<0.000]$; thus, univariate follow-up comparisons between the three drinking groups were examined by using the Bonferroni post hoc test, which adjusts the observed significance level when multiple group comparisons are made. As can be seen in Table 3, there were no significant differences among the three drinking groups in marital status, CES-D, number of previous children, years of school, or racial/ethnic group. Significant drinking group differences were found in weeks of pregnancy, number of cigarettes per day, and maternal age. Women in the higher risk group were significantly earlier in their pregnancy (mean weeks $=20.8, \mathrm{SD}=10.6$ ) than the low-risk group (mean weeks $=26.9, \mathrm{SD}=10.2)$. Women in the higher risk group smoked significantly more cigarettes per day (mean $=0.75, \mathrm{SD}=1.2)$ than either the low-risk (mean $=0.35$, $\mathrm{SD}=0.92$ ) or no alcohol use group (mean $=0.24, \mathrm{SD}=$ $0.72)$. Low-risk drinking women were significantly older $($ mean $=29.9, \mathrm{SD}=4.6)$ than those who did not drink 
while pregnant $($ mean $=28.5, \mathrm{SD}=5.4)$, with no age differences found for the high-risk group.

\section{Factors Associated With Elevated TWEAK}

Among the higher risk drinkers (i.e., those who reported one or more drinks per week and/or at least one binge episode during pregnancy), $29.4 \%$ scored above the cutoff on the TWEAK. Given the moderate overlap of these two risk indicators, factors associated with elevated TWEAK were also examined by using logistic regression analyses. The overall model was found to be significant $\left[\chi^{2}(10)=\right.$ 99.4, $p<0.000$ ] with the following predictors included in the model: weeks of pregnancy, number of previous children, cigarette use during pregnancy, years of school completed, maternal age, marital status, race, CES-D score, and physician mention of alcohol use. Marital status, education, and number of weeks pregnant were not found to be significant predictors of TWEAK; however, number of weeks pregnant approached significance $(\beta=-0.02, p=$ 0.07 , odds ratio $=0.98)$. Maternal age $(\beta=-0.09$, odds ratio $=0.91, p<0.001)$ and number of previous children $(\beta$ $=-0.47$, odds ratio $=0.63, p<0.001$ ) were found to have significant negative coefficients, indicating that the odds of scoring above the cutoff on the TWEAK diminish with older maternal age and greater number of previous children. Smoking $(\beta=0.26, p=0.02)$, CES-D $(\beta=0.05, p=$ $0.00)$, race $(\beta=1.3, p=0.00)$, and alcohol use risk status $(\beta=0.46, p=0.02)$ were found to have significant positive coefficients, indicating that the odds of scoring above the cutoff on the TWEAK are 1.3 times higher as cigarettes per day increase, 1.1 times higher as CES-D score increases, 3.7 times higher for Caucasians than for African Americans, and 1.6 times higher for those in a higher alcohol use risk group.

\section{DISCUSSION}

The purpose of this study was to demonstrate the feasibility of screening in obstetrics settings, to identify rates and correlates of alcohol use in a large, demographically representative sample of pregnant women across a number of obstetrics clinics, extending previous studies of single or high-risk settings. The study found that $15.1 \%(n=168)$ of women in the sample reported using any alcohol while pregnant. This rate is consistent with the 1995 survey conducted by Ebrahim et al. (1998), who found that $15.3 \%$ reported any alcohol consumption in the past month. Most women who drank while pregnant in this sample indicated that they drank one standard drink or fewer per occasion. Our higher risk group was defined as those having one or more standard drinks per week and/or at least one binge episode during pregnancy, and this group comprised 3\% of the total sample screened. Jacobson and Jacobson (1994) reported some decrements in neurobehavioral outcomes of infants prenatally exposed to "very light" (defined as 0.02 3.49 standard drinks per week) levels. Among women in our sample who reported prenatal alcohol use, $6.1 \%$ of the women reported one or more occasions of consuming five or more standard drinks, a level that has been found to relate to functional impairments in infants (Jacobson and Jacobson, 1999). In addition, $13 \%$ of women in the sample scored above the cutoff score on the TWEAK, which has been found to have a sensitivity of 0.91 in identifying highrisk drinkers (Russell, 1994). Interventions targeting these higher risk groups of women, therefore, may effectively prevent developmental abnormalities resulting from prenatal alcohol exposure (selective prevention). Different screening indexes seemed to result in differential detection of risk. For example, a minority of women in the higher risk group (based on direct questioning about alcohol use while pregnant) scored above the cutoff on the TWEAK, which asked about the year before pregnancy (29\%). Therefore, it may be useful to use multiple methods of screening (both direct and indirect) to capture a broader group of women who may be at risk for negative outcomes.

These findings support the feasibility and utility of routine screening in women seeking prenatal care. The screening measures took less than $10 \mathrm{~min}$ to complete and, in most cases, were easily completed while the patients waited for their appointments. The response rate was high (92\%), indicating the acceptability of the procedures and questions included in the instrument. Screening results may be easily tabulated and attached to the charts prior to discussion with their physicians. Research assistants were used to hand out the screening questionnaires to women, which potentially limits the generalizability to routine clinic screening by clerical or clinical staff. However, these assistants required very little training and were incorporated into normal clinic procedures as much as possible. Although these findings demonstrated a significant positive association between provider discussion of alcohol use and TWEAK, only $54.5 \%$ of those who drank reported that their health care provider had talked with them about alcohol use. Routine screening may substantially improve detection rates, which may be coupled with brief interventions. Brief advice by physicians has been found to significantly reduce high-risk drinking behavior in childbearing age women in primary care (Fleming et al., 1997; Manwell et al., 2000).

Multivariate analyses found that earlier stage of pregnancy and greater cigarette use were significantly associated with higher risk alcohol use while pregnant. Consistent with other studies, these results suggest that riskier drinking tapers off as pregnancy progresses (Little and Streissguth, 1978; Waterson and Murray-Lyon, 1989; Weiner et al., 1983). Cigarette use during pregnancy appears to be a robust predictor of higher risk drinking, as demonstrated in this and other studies (Gladstone et al., 1997; Sokol et al., 1981; Svikis et al., 1997; Waterson and Murray-Lyon, 1989). Prenatal clinical encounters should consistently include assessment of tobacco use both as an independent risk to the infant as well as an indicator for co-occurring high-risk alcohol use. Although older maternal age was related to 
alcohol use in pregnancy, it did not predict higher risk drinking as indicated by higher levels of alcohol use or by scoring above the cutoff on the TWEAK.

In this study, race and education were not predictive of higher risk alcohol use during pregnancy, as was found in at least two other studies (Chasnoff et al., 1990; Waterson and Murray-Lyon, 1989). However, other studies have found racial differences in women who continue to drink throughout pregnancy (Smith et al., 1987) and women who binge drink during pregnancy (Gladstone et al., 1997). Given our low rates of higher risk drinking $(n=31)$ and the crosssectional design of the study, our sample may not have had sufficient numbers of other racial groups who were higher risk alcohol users or multiple assessments necessary to detect racial differences. Caucasian women in our sample were significantly more likely to score above the cutoff on the TWEAK than African American women. Previous research suggests that Caucasian women may be more likely to drink at risky levels during pregnancy (Day et al., 1993; Gladstone et al., 1997; Hans, 1999). Although previous studies have found marital status to be related to prenatal drinking (Centers for Disease Control and Prevention, 1995), this study found no such association.

A concern with this study is the potential for compromised generalizability resulting from informing women that their responses would be kept confidential from their healthcare provider, which would not be the case in routine clinical screening. It is possible that women would be less willing to report alcohol use during pregnancy if they knew that their providers would be given the information. However, in a number of brief alcohol intervention trials, providers have been given information about alcohol use. Fleming and Barry (1991), in large screening and brief alcohol intervention (Fleming et al., 1997) studies that included women of childbearing age, found similar at-risk drinking prevalence rates indicating that primary care patients provide relatively accurate and consistent information on drinking patterns whether or not providers could be given the information. Also, maternal self-report of alcohol use in pregnancy has been found to be relatively accurate (Chang et al., 1999a). A positive response of any alcohol use may provide the opportunity to give clear prevention messages and the opportunity to prevent future harm.

Possible bias may also have been introduced by our $8 \%$ refusal rate for the screening questionnaire. Although we were unable to systematically assess reasons for refusal, anecdotal observations reveal that women in an irritable mood, those who were accompanied by multiple other children to their appointment, and those who were themselves medical professionals or researchers were more likely to refuse. Future studies should systematically assess factors that are associated with unwillingness to complete screening measures in a healthcare setting so that possible confounding factors may be evaluated and controlled. We do not believe that provision of the educational booklet (which outlines all aspects of a healthy pregnancy including the possible effects of alcohol and tobacco use) affected responses to the screening measures because the booklet was handed to the women after completion of all study procedures (i.e., after participants completed the screening measure and consent form).

It is also important to note that these data are crosssectional. It is likely that different predictors for alcohol use throughout pregnancy with repeated collection of alcohol use data may have been found (such as racial and educational differences). The measures used in this study were solely screening measures. Future studies should include a thorough diagnostic assessment of alcohol use and alcohol diagnoses (such as the time line follow back and diagnostic interview schedule).

Healthcare provider recognition and advice regarding alcohol use during pregnancy are likely to have a substantial impact on prevention of negative infant outcomes. This study demonstrated that screening in busy obstetrics clinics is feasible and acceptable to women, that it may be optimal to use both indirect and direct measures of alcohol use, and that assessments of alcohol use should be conducted throughout pregnancy because decreasing and stopping use at any phase of the pregnancy can be helpful both to the patient and to her developing fetus (Bresnahan et al., 1992; Wilsnack, 1996).

\section{REFERENCES}

Abel EL (1995) An update on incidence of FAS: FAS is not an equal opportunity birth defect. Neurobehav Toxicol 17:437-443.

Babor TF, Stephens RS, Marlatt GA (1987) Verbal report methods in clinical research on alcoholism: response bias and its minimization. $\mathrm{J}$ Stud Alcohol 48:410-424.

Barry KL (1999) Treatment Improvement Protocol: Brief Alcohol Interventions and Therapies in Substance Abuse Treatment. Center for Substance Abuse Treatment, Substance Abuse and Mental Health Services Administration, Rockville, MD.

Bradley KA, Boyd-Wickizer J, Powell SH, Burman ML (1998) Alcohol screening questionnaires in women: a critical review. JAMA 280:166171.

Bresnahan K, Zuckerman B, Cabral H (1992) Psychosocial correlates of drug and heavy alcohol use among pregnant women at risk for drug use. Obstet Gynecol 80:976-980.

Brown SS (1989) Drawing women into prenatal care. Fam Plann Perspect 21:73-80.

Centers for Disease Control and Prevention (1995) Sociodemographic and behavioral characteristics associated with alcohol consumption during pregnancy-US. MMWR Morb Mortal Wkly Rep 14:261-264.

Chang G, Goetz A, Wilkens-Haug L, Berman M (1999a) Prenatal alcohol consumption: self versus collateral report. J Subst Abuse Treat 17:8589.

Chang G, Wilkens-Haug L, Berman M, Goetz A (1999b) Brief intervention for alcohol use in pregnancy: a randomized trial. Addiction 94:111.

Chasnoff IJ, Landress HJ, Barrett ME (1990) The prevalence of illicit drug or alcohol use during pregnancy and discrepancies in mandatory reporting in Pinellas County, Florida. N Engl J Med 322:1202-1206.

Corcoran K, Fisher J (1987) Measures for Clinical Practice: A Sourcebook. Free Press, New York.

Day NL, Cottreau CM, Richardson GA (1993) The epidemiology of alcohol, marijuana, and cocaine use among women of childbearing age and pregnant women. Clin Obstet Gynecol 36:232-245. 
Ebrahim SH, Luman ET, Floyd RL, Murphy CC, Bennett EM, Boyle CA (1998) Alcohol consumption by pregnant women in the United States during 1988-1995. Obstet Gynecol 92:187-192.

Ewing, JA (1984) Detecting alcoholism: the CAGE questionnaire. JAMA 252:1905-1907.

Fleming M, Barry KL (1991) A three-sample test of a masked alcohol screening questionnaire. Alcohol Alcohol 26:81-91.

Fleming MF, Barry KL, Manwell LB, Johnson K, London R (1997) Brief physician advice for problem alcohol drinkers: a randomized controlled trial in community-based primary care practices. JAMA 277:1039-1045.

Gladstone J, Levy M, Nulman I, Koren G (1997) Characteristics of pregnant women who engage in binge alcohol consumption. Can Med Assoc J 156:789-794.

Halmesmaki E (1988) Alcohol counseling of 85 pregnant problem drinkers: effect on drinking and fetal outcomes. J Obstet Gynaecol 95:243247.

Hankin J (1994) FAS prevention strategies: passive and active measures. Alcohol Health Res World 18:74-81.

Hankin J, McCaul ME, Heussner J (2000) Pregnant, alcohol-abusing women. Alcohol Clin Exp Res 24:1276-1286.

Hans SL (1999) Demographic and psychosocial characteristics of substance-abusing pregnant women. Clin Perinatol 26:55-74.

Heller J, Anderson HR, Bland JM, Brooke OG, Peacock JL, Stewart CM (1988) Alcohol in pregnancy: patterns and association with socioeconomic, psychological, and behavioral factors. Br J Addict 83:541551.

Husaini BA, Neff JA, Harrington JB, Hughe MD, Stone RH (1980) Depression in rural communities: validating the CES-D scale. J Commun Psychol 8:20-27.

Jacobson JL, Jacobson SW (1994) Prenatal alcohol exposure and neurobehavioral development. Alcohol Health Res World 23:30-36.

Jacobson JL, Jacobson SW (1999) Drinking moderately and pregnancy: effects on child development. Alcohol Health Res World 23:25-30.

Kelly RH, Zatzick DF, Anders TF (2001) The detection and treatment of psychiatric disorders and substance abuse among pregnant women cared for in obstetrics. Am J Psychiatry 158:213-219.

Larsson G (1983) Prevention of fetal alcohol effects: an antenatal program for early detection of pregnancies at risk. Acta Obstet Gynecol Scand 62:171-178.

Little RE, Streissguth AP (1978) Drinking during pregnancy in alcoholic women. Alcohol Clin Exp Res 2:179-183.

Little RE, Young A, Streissguth AP, Uhl CN (1984) Preventing fetal alcohol effects: effectiveness of a demonstration project, in Mechanisms of Alcohol Damage in Utero (Porter R, O'Connor M, Whelan J, eds.), pp. 254-274. CIBA Foundation Symposium, London.

Manwell LB, Fleming MF, Mundt MP, Stauffacher EA, Barry KL (2000) Treatment of problem alcohol use in women of childbearing age: results of a brief intervention trial. Alcohol Clin Exp Res 24:1517-1524.
May PA (1996) Research issues in the prevention of fetal alcohol syndrome and alcohol related birth defects. Patterns and trends in women's drinking: recent findings and some implications for prevention. Women and Alcohol: Issues for Prevention Research. National Institute on Alcohol Abuse and Alcoholism Monograph No. 32, DHHS publication no 96-3817. National Institute on Alcohol Abuse and Alcoholism, Bethesda, MD.

National Pregnancy and Health Survey (1996) Drug Abuse Among Women Delivering Live Births: 1992. NIH publication 96-3819. DHHS, NIDA, Rockville MD.

Radloff LS (1977) The CES-D scale: a self-report depression scale for research in the general population. Appl Psychol Meas 1:385-401.

Russell M (1994) New assessment tools for risk drinking during pregnancy. Alcohol World 18:55-61.

Santor DA, Zuroff DC, Mongrain M, Fielding A (1997) Validating the McGill revision of the depressive experiences questionnaire. J Pers Assess 69:164-182.

Saunders JB, Aasland OG, Amudsen A, Grant M (1993) WHO collaborative project on early detection of persons with harmful alcohol consumption. Addiction 88:349-362.

Smith IE, Lancaster JS, Moss-Wells S, Coles CD, Falek A (1987) Identifying high risk pregnant drinkers: biological and behavioral correlates of continuous heavy drinking during pregnancy. J Stud Alcohol 48:304309.

Sokol RJ, Miller SI, Debanne S, Golden N, Collins G, Kaplan J, Martier S (1981) The Cleveland NIAAA Prospective Alcohol-in-Pregnancy Study: the first year. Neurobehav Toxicol Teratology 3:203-209.

Stratton K, Howe C, Battaglia F (eds) (1996) Institute of Medicine Summary: Fetal Alcohol Syndrome. National Academy Press, Washington, DC.

Svikis D, Henningfield J, Gazaway P, Huggins G, Sosnow K, Hranicka J, Harrow C, Pickens R.(1997) Tobacco use for identifying pregnant women at risk of substance abuse. J Reprod Med May;42(5):299-302

U.S. Census Bureau (2000) Michigan Census Data.

Waterson EJ, Murray-Lyon IM (1989) Drinking patterns among women attending an antenatal clinic during pregnancy. Alcohol Alcohol 24: 163-173.

Weiner L, Morse BA (1996) Fetal alcohol syndrome: a framework for successful prevention, in Alcohol, Pregnancy and the Developing Child (Spohr HL, Steinhausen HC eds). Cambridge University Press, Cambridge, UK.

Weiner L, Rosett HL, Edelin KC, Alpert JJ, Zuckerman B (1983) Alcohol consumption by pregnant women. Obstet Gynecol 61:6-12.

Wilsnack SC (1996) Patterns and trends in women's drinking: recent findings and some implications for prevention. Women and Alcohol: Issues for Prevention Research. National Institute on Alcohol Abuse and Alcoholism Monograph No. 32. DHHS publication no. 96-3817. National Institute on Alcohol Abuse and Alcoholism, Bethesda, MD. 